\section{Coagulation disorders and the IPF puzzle}

\author{
David B Coultas
}

While idiopathic pulmonary fibrosis (IPF) remains a frustrating mystery for patients and their physicians, growing evidence from a number of different sources provides potentially clinically relevant clues to the puzzle of this largely untreatable and highly fatal disorder. ${ }^{12}$ Answers to frequently asked questions from patients of 'why me?' 'what is the cause?' 'what can be done?' and 'what can I expect?' are gradually emerging from studies of pathogenesis, clinical trials and epidemiological investigations.

For over three decades, the majority of IPF research has focused on pathogenesis and clinical trials of various therapeutic agents. ${ }^{2}$ Available evidence on pathogenic mechanisms suggests that pulmonary fibrosis results from a number of exposures that cause alveolar epithelial injury (eg, cigarette smoke, asbestos fibres, viruses) followed by activation of a complex set of abnormal, genetically determined biological pathways that result in fibrosis. ${ }^{3}$ Altering the course of the disease remains a challenge partly because of the complexity of the pathogenesis of IPF, and time needed for discovery and clinical trials of new agents to determine efficacy. Moreover, despite the growing number of clinical trials among patients with symptomatic IPF the results have been disappointing. Therefore, parallel research approaches are needed on interventions to prevent the occurrence (ie, primary prevention) or progression of subclinical pulmonary fibrosis (ie, secondary prevention).

For any disease, the ultimate goal is primary prevention through elimination of exposures that cause the disease. While epidemiological evidence suggests this may be a possible approach for IPF, there are also major challenges. Exposure to a number of environmental and occupational agents may cause IPF, ${ }^{4}$ and control of these exposures offers the potential to prevent the development of IPF. For example, based on the prevalence of smoking and risk of IPF associated with smoking an estimated $49 \%$ of IPF cases

Correspondence to Dr David B Coultas, Department of Medicine, The University of Texas Health Science Center-Tyler, Tyler, TX 75708 USA; david.coultas@ uthct.edu may be prevented through elimination of this exposure. However, the relatively low occurrence of IPF and challenges with control of multiple exposures limits the feasibility of conducting trials of primary prevention. Interventions may become feasible with the use of biomarkers of IPF to identify high-risk individuals. ${ }^{5}$

Secondary prevention or early detection combined with interventions to prevent progression of subclinical disease may be accomplished through advances in diagnostic sensitivity and accuracy with highresolution CT (HRCT) scans and other biomarkers. ${ }^{5-7}$ The feasibility of this approach is supported by recent findings of subclinical interstitial lung abnormalities by HRCT scanning among several different populations. ${ }^{8} 9$ While the relationship between these abnormalities and IPF remains to be established, clinical and radiographic characteristics suggest patterns similar to IPF. For example, Doyle et $a l^{10}$ found that among smokers $8 \%$ had interstitial lung abnormalities and among these $75 \%$ had a subpleural or mixed pattern consistent with the radiographic distribution of IPF. Moreover, subclinical interstitial lung abnormalities were associated with clinically relevant impairment of spirometry and 6 min walking distance (Doyle et $a l^{10}$ ). In addition to HRCT for early detection the use of biomarkers has the potential to detect high-risk individuals with subclinical disease. 579

Navaratnam et $a l^{11}$ add to the epidemiological evidence relevant to biomarkers and identification of high-risk individuals. They conducted a population-based, casecontrol study in the Greater Trent Region of the UK to examine the risk of IPF associated with a prothrombotic state. The investigators enrolled 211 incident cases of clinically stable patients with IPF diagnosed by a respiratory physician. To confirm the diagnosis and further categorise cases as definite or probable, HRCT scans were reviewed by two experienced thoracic radiologists. Age-matched and gender-matched population-based controls $(n=256)$ were identified from 10 primary care practices from the same geographic region as the patients with IPF. The thrombotic state was characterised with a panel of 11 laboratory tests that included seven inherited and four acquired clotting defects. In a multivariate model adjusting for age, sex, smoking status and highly sensitive C-reactive protein (CRP), the risk (OR) of IPF associated with at least one abnormality of coagulation was substantially increased $(\mathrm{OR}=4.78$ (95\% CI 2.93 to 7.80)). Moreover, there was a doseresponse increase in the risk of IPF associated with the number of thrombotic abnormalities. Other clinically relevant findings included associations between prothrombotic state and greater impairment of forced vital capacity and diffusing capacity for carbon monoxide (DLCO) and increased mortality.

The investigators carefully designed and executed this study to minimise potential biases and strengthen both external and internal validity. Specific study design characteristics and results that support robust external validity of this investigation include (1) enrolment of a broadly representative sample of patients with newly diagnosed IPF from different types of hospitals, including five teaching and eight district general hospitals, (2) high response rate $(86 \%)$ of eligible patients, (3) control subjects from the same geographic region and (4) patient characteristics of age, gender, distribution of severity of impairment and mortality similar to other investigations and (5) an increased risk of smoking similar to previous studies. ${ }^{4}$

In addition, several study design characteristics support strong internal validity. These characteristics include (1) minimisation of potential diagnostic misclassification of IPF by the use of standardised diagnostic criteria, independent verification by experienced thoracic radiologists and stratified analysis of definite and probable cases, (2) use of a panel of 11 objective measures of a prothrombotic state, (3) control of major potential confounding factors, including age, gender, smoking and highly sensitive CRP and (4) sufficient power to examine dose-response relationships.

While the results of this investigation establish a strong association between a prothrombotic state and IPF, alone they do not prove causation. However, when evaluated in the context of other epidemiological and biological evidence these results provide a compelling case for a causal role of coagulation disorders in the development of IPF.

Causal inference is a complex process that is based on the quality of evidence from multiple sources and an evaluation of that evidence using nine criteria, including magnitude of risk, doseresponse, consistency, plausibility, coherence, temporality, analogy, experiment 
and specificity. ${ }^{12}$ The magnitude of risk of IPF associated with the prothrombotic state in this study is large (adjusted $\mathrm{OR}=4.78$ ), which makes alternative explanations for the association of chance and confounding unlikely. Moreover, the large risk also suggests that coagulation defects have a major effect on the fibrotic process that is further supported by the dose-response relationship of an incremental increase in risk of IPF associated with an increase in the number of coagulation defects. The calculation of the population attributable fraction from the prevalence of prothrombotic state among controls (46.5\%) and magnitude of risk $(\mathrm{OR}=4.78)$ suggests that control of the prothrombotic state has the potential to prevent $60 \%$ of IPF cases. Finally, the results of this study are consistent with three previous investigations that used clinical diagnoses of venous thromboembolism to estimate the risk of IPF. ${ }^{13-15}$

Of the other criteria used to assess causation, four are most relevant to evidence on biological mechanisms, including plausibility, coherence, temporality and analogy. The plausibility of a causal link between prothrombotic state and IPF is strong and coherent with current understanding of the pathogenesis of IPF. ${ }^{16}$ Moreover, there is an analogous sequence of events and outcomes with exposure to asbestos and resultant asbestosis, which is indistinguishable from the pathology of IPF.

Of the original nine criteria, specificity and experiment have little relevance to chronic diseases such as IPF or there is limited available evidence, respectively. A specific one-to-one link between the prothrombotic state and pulmonary fibrosis is not expected based on our current understanding of the complex pathways involved in the pathogenesis of $\mathrm{IPF}^{3}{ }^{5}$ Finally, experimental evidence from clinical trials of anticoagulation in patients with IPF has provided conflicting results ${ }^{16-18}$ and further research is need to address the experimental criterion.

Overall, results from this epidemiological investigation reported by Navaratnam et $a l^{11}$ on the risk of IPF associated with a prothrombotic state provide another piece to the puzzle of IPF, and when combined with other lines of evidence on biological mechanisms provide strong support for the role of coagulation disorders in the causation of IPF. Furthermore, these results suggest that biomarkers of coagulation, along with other omics-based biomarkers ${ }^{5}$ may be useful to identify high-risk individuals to target for investigations of primary and secondary prevention and to focus research on novel agents for secondary and tertiary prevention.

Acknowledgements I thank Dr David Griffith for review and helpful comments.

\section{Competing interests None.}

Provenance and peer review Commissioned; internally peer reviewed.

To cite Coultas DB. Thorax 2014;69:203-204.

Published Online First 16 September 2013

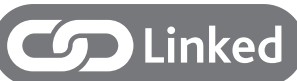

http://dx.doi.org/10.1136/thoraxjnl-2013-203740

Thorax 2014;69:203-204

doi:10.1136/thoraxjnl-2013-204245

\section{REFERENCES}

1 Raghu G, Collard HR, Egan JJ, for the ATS/ERS/JRS/ ALAT Committee on Idiopathic Pulmonary Fibrosis. An official ATS/ERS/JRS/ALAT statement: idiopathic pulmonary fibrosis: evidence-based guidelines for diagnosis and management. Am I Respir Crit Care Med 2011;183:788-824.
2 Richeldi L, Collard HR, du Bois RM, et al. Mapping the future for pulmonary fibrosis: report from the 17th International Colloquium on Lung and Airway Fibrosis. Eur Respir J 2013;42:230-8.

3 Wuyts WA, Agostini C, Antoniou KM, et al. The pathogenesis of pulmonary fibrosis: a moving target. Eur Respir J 2013;41:1207-18.

4 Taskar VS, Coultas DB. Is idiopathic pulmonary fibrosis an environmental lung disease? Proc Am Thorac Soc 2006:3:293-8.

5 Maher TM. PROFILEing idiopathic pulmonary fibrosis: rethinking biomarker discovery. Eur Respir J 2013;128:148-52.

6 Lederer DJ. Secondary prevention of idiopathic pulmonary fibrosis: catching the horse still in the barn. Am J Respir Crit Care Med 2012;185:697-99.

7 Herazo-Maya JD, Kaminski N. Personalized medicine: applying 'omics' to lung fibrosis. Biomark Med 2012:6:529-40

8 Doyle TJ, Hunninghake GM, Rosas IO. Subclinical interstitial lung disease. Why you should care. Am J Respir Crit Care Med 2012;185:1147-53.

9 Hunninghake GM, Hatabu H, Okajima Y, et al. MUC5B promoter polymorphism and interstitial lung abnormalities. N Eng/ J Med 2013;368:2192-200.

10 Doyle TJ, Washko GR, Fernandez IE, et al. Interstitial lung abnormalities and reduced exercise capacity. $A m$ J Respir Crit Care Med 2012;185:756-62.

11 Navaratnam V, Fogarty AW, McKeever T, et al. Presence of a prothrombotic state in people with idiopathic pulmonary fibrosis: a population-based case-control study. Thorax 2014:69:207-15.

12 Hill $A B$. The environment and disease: association or causation? Proc $R$ Soc Med 1965:58:295-300.

13 Hubbard RB, Smith C, Le Jeune I, et al. The association between idiopathic pulmonary fibrosis and vascular disease: a population-based study. Am J Respir Crit Care Med 2008;178:1257-61.

14 Sode BF, Dahl M, Nielsen SF, et al. Venous thromboembolism and risk of idiopathic interstitial pneumonia: a nationwide study. Am J Respir Crit Care Med 2010;181:1085-92.

15 Sprunger DB, Olson AL, Huie TJ, et al. Pulmonan fibrosis is associated with an elevated risk of thromboembolic disease. Eur Respir J 2012;39:125-32.

16 Mercer PF, Chambers RC. Coagulation and coagulation signaling in fibrosis. Biochimica et Biophysica Acta 2013;1832:1018-27.

17 Kubo H, Nakayama K, Yanai M, et al. Anticoagulant therapy for idiopathic pulmonary fibrosis. Chest 2005; 128:1475-82.

18 Noth I, Anstrom KJ, Calvert SB, et al. A placebo-controlled randomized trial of warfarin in idiopathic pulmonary fibrosis. Am J Respir Crit Care Med 2012;186:88-95. 